\title{
QUASILOCAL FORMALISM FOR DILATON GRAVITY WITH YANG-MILLS FIELDS
}

\author{
Jolien D. E. Creighton and Robert B. Mann
}

25 July, 1995

\begin{abstract}
We present a quasilocal formalism, based on the one proposed by Brown and York, for dilaton gravity with Yang-Mills fields. For solutions possessing sufficient symmetry, we define conserved quantities such as mass, angular momentum, and charge. We also present a micro-canonical action and use it to arrive at a quasilocal version of the first law of thermodynamics for static systems containing a black hole.
\end{abstract}

\section{INTRODUCTION}

Suppose a gravitating system ${ }^{1}$ is surrounded by some spatial surface. On this surface, it is possible to define quantities that will yield information about the system contained. These quantities we call quasilocal quantities. In the past it has been the practice to determine analogous quantities in an asymptotic limit. However, this procedure must be adapted for the specific asymptotic behaviour of the spacetime under consideration; it is unclear how to proceed except when the spacetime is asymptotically flat. Furthermore, when we study the thermodynamics of a gravitating system, the quasilocal surface takes on an important physical significance as the thermodynamic wall containing the system. Thus, we find it important to develop a formalism for constructing quasilocal quantities on the boundary of a gravitating system, as such quantities can then be identified directly as the thermodynamic variables of the system.

An excellent quasilocal formalism was developed by Brown and York [Brown and York (1993a)] for General Relativity. In this formalism, a surface stress energy momentum tensor was constructed by a functional analysis of the action for General Relativity, and, from this tensor, a quasilocal energy can be found. In addition, for spacetimes possessing sufficient symmetry, a conserved mass (which differs, in general, from the quasilocal energy) and angular momentum can be defined. In their following paper [Brown and York (1993b)], they used the Feynman path integral technique of statistical mechanics to demonstrate the connection between canonical and thermodynamic conjugacy. They showed how to construct a "microcanonical" action for which the "extensive" variables are fixed on the boundary of the system. From this form of the action, the entropy and the first law of thermodynamics (at the zeroth order of quantum corrections) follow easily.

1991 Mathematics Subject Classification. Primary 83C40; Secondary 83C57, 81T13.

This work was supported by NSERC, Canada.

${ }^{1}$ In this paper, we shall assume a four dimensional spacetime for definiteness. 
Our purpose is to extend this formalism to gravity theories involving a dilaton. In addition, we will include a Yang-Mills field in our analysis to demonstrate the rôles of matter in the formalism. This paper is a summary of the formalism; a more complete discussion is given in our recent paper [Creighton and Mann (1995)].

\section{The Quasilocal Formalism}

Assume that the region of spacetime under consideration, $\mathcal{M}$ (the "gravitating system"), is topologically the direct product of a spacelike hypersurface, $\Sigma$, with a real interval. The boundary $\partial \Sigma$ is the quasilocal surface that surrounds the system, and $\mathcal{T}$ is the history of this surface. We take our dynamical fields to be the spacetime metric, $g_{\mu \nu}$, a scalar field we call the dilaton, $\Phi$, and a Yang-Mills potential $\mathfrak{A}_{\mu}{ }^{\mathfrak{a}}$. The gauge covariant derivative operator is $\left(\mathfrak{D}_{\mu}\right)^{\mathfrak{a}} \mathfrak{b}=\nabla_{\mu} \delta^{\mathfrak{a}} \mathfrak{b}+\mathfrak{f}^{\mathfrak{a}}{ }_{\mathfrak{b c}} \mathfrak{A}_{\mu}{ }^{\mathfrak{c}}$ where $\mathfrak{f}^{\mathfrak{a}} \mathfrak{b} \mathfrak{c}$ are the structure constants of the gauge group. ${ }^{2}$ The action we take is:

$$
\begin{aligned}
S=\int_{\mathcal{M}} & d^{4} x \sqrt{-g}\left(D(\Phi) R(g)+H(\Phi)(\nabla \Phi)^{2}+V(\Phi)-\frac{1}{4} W(\Phi) \mathfrak{F}^{\mu \nu}{ }_{\mathfrak{a}} \mathfrak{F}_{\mu \nu}{ }^{\mathfrak{a}}\right) \\
& -2 \int_{\mathcal{T}} d^{3} x \sqrt{-\gamma} D(\Phi) \operatorname{tr}(\Theta)-2 \int_{\Sigma} d^{3} x \sqrt{h} D(\Phi) \operatorname{tr}(K) .
\end{aligned}
$$

Here, $R(g)$ is the Ricci scalar of the metric $g_{\mu \nu}$ and $\mathfrak{F}_{\mu \nu}{ }^{\mathfrak{a}}$ is the curvature of the gauge covariant derivative operator. The induced metrics on $\mathcal{T}$ and $\Sigma$ are $\gamma_{i j}$ and $h_{i j}$ respectively, while the extrinsic curvatures of these surfaces are $\Theta_{i j}$ and $K_{i j}$ respectively. The functions $D(\Phi), H(\Phi), V(\Phi)$, and $W(\Phi)$ are arbitrary functions of the dilaton alone (and none of its derivatives) that specify the theory. ${ }^{3}$

Under variations of the field configurations for which the boundary configurations are fixed the induced variation in the action of equation (1) is minimized when the equations of motion are satisfied. Under more arbitrary variations, surface terms proportional to the variations of the fields on the boundaries will appear along with the equation of motion terms. The coefficients of the boundary variations of the fields are identified as the momenta conjugate to those surface fields. The phase space on $\Sigma$ is given by $\left\{\left(p^{i j}, h_{i j}\right),\left(\mathfrak{P}^{i}{ }_{\mathfrak{a}}, \mathfrak{A}_{i}^{\mathfrak{a}}\right),(P, \Phi)\right\}$ so that, for example, $\pi^{i j}=-\left(\delta S / \delta h_{i j}\right)_{c \ell}$ (where the "cl" indicates evaluation on a classical solution). There will also be momenta conjugate to the fields on the $\mathcal{T}$ boundary; its phase space coördinates are $\left\{\left(\pi^{i j}, \gamma_{i j}\right),\left(\mathfrak{K}_{\mathfrak{a}}^{i}, \mathfrak{U}_{i}^{\mathfrak{a}}\right),(\Pi, \phi)\right\}$. (We have written the gauge and dilaton fields on $\mathcal{T}$ as $\mathfrak{U}_{i}{ }^{\mathfrak{a}}$ and $\phi$ respectively.)

Our primary interest will be in the $\mathcal{T}$ boundary which is the history of our quasilocal surface. Parameterize the time interval with $t$ whose corresponding affine vector is $t^{\mu}=N u^{\mu}+N^{\mu}$ where $u^{\mu}$ is the unit normal to the leaves, $\Sigma_{t}$, of the foliation, and $N$ and $N^{\mu}$ are the lapse and shift vectors. On the quasilocal surfaces, $\partial \Sigma_{t}$, there are induced metrics $\sigma_{i j}=\gamma_{i j}+u_{i} u_{j}$. We can decompose the fields and conjugate momenta on $\mathcal{T}$ into portions parallel to the unit normals, $u^{i}$ (which we assume lie along $\mathcal{T}$ ), and projections onto the surfaces $\partial \Sigma$. The metric, $\gamma_{i j}$, is thus decomposed into functions of the lapse, the shift, and the induced metric $\sigma_{a b}$ in the usual way. The gauge field, $\mathfrak{U}_{i}^{\mathfrak{a}}$ is decomposed into a scalar, $\mathfrak{V}$, and a vector on $\partial \Sigma$,

\footnotetext{
${ }^{2}$ From these structure constants, we are able to define a Killing metric that will allow us to raise and lower gauge group indices.

${ }^{3}$ The action functional can also posess an arbitrary functional of the boundary fields, but we shall ignore this here.
} 
$\mathfrak{W}_{a}{ }^{\mathfrak{a}}$. Similarly we decompose the momenta. Define, on $\partial \Sigma, \mathcal{E}=\sqrt{\sigma} u_{i} u_{j} \tau^{i j}$, and, $\mathcal{S}^{a b}=\sqrt{\sigma} \sigma_{i}^{a} \sigma_{j}^{b} \tau^{i j}$ as the quasilocal surface energy and stress density respectively. Here, $\tau^{i j}=2 \pi^{i j} / \sqrt{-\gamma}$ is the surface stress energy momentum tensor on $\mathcal{T}$. Similarly, define $\mathcal{Q}_{\mathfrak{a}}=\sqrt{\sigma} W(\Phi) n_{i} \mathfrak{E}_{\mathfrak{a}}^{i}$ and $\mathcal{I}_{a}{ }^{\mathfrak{a}}=\sqrt{\sigma} W(\Phi) \epsilon_{a}{ }^{i j} n_{i} \mathfrak{B}_{j}{ }^{\mathfrak{a}}$ as the quasilocal Yang-Mills surface charge and current densities respectively. Here, $n^{i}$ is the unit normal to $\Sigma$ and $\mathfrak{E}_{i}{ }^{\mathfrak{a}}$ and $\mathfrak{B}_{i}{ }^{\mathfrak{a}}$ are the usual electric and magnetic (spatial) vectors constructed from the Yang-Mills field strength. The surface momentum density is given by $\mathcal{J}^{a}=-\sqrt{\sigma} u_{i} \sigma_{j}^{a} \tau^{i j}+\mathcal{Q}^{\mathfrak{a}} \mathfrak{W}^{a}{ }_{\mathfrak{a}}$; note that it includes an EMF term from the Yang-Mills field. Finally, a surface dilaton density is given by $\mathcal{F}=\Pi / N$. Explicit expressions for these quantities are given elsewhere [Creighton and Mann(1995)].

With these definitions, the variation of the action on the boundary $\mathcal{T}$ is

$$
\left.\delta S\right|_{\mathcal{T}}=\int_{\mathcal{T}} d^{3} x\left(-\mathcal{E} \delta N+\mathcal{J}_{a} \delta N^{a}-\mathcal{Q}_{\mathfrak{a}} \delta\left(N \mathfrak{V}^{\mathfrak{a}}\right)+N\left(\frac{1}{2} \mathcal{S}^{a b} \delta \sigma_{a b}+\mathcal{I}^{a}{ }_{\mathfrak{a}} \delta \mathfrak{W}_{a}{ }^{\mathfrak{a}}+\mathcal{F} \delta \phi\right)\right)
$$

Any variable that is a function of the phase space coördinates (on $\Sigma$ ) only is called an extensive variable. The variables $\mathcal{E}, \mathcal{J}_{a}, \mathcal{Q}_{\mathfrak{a}}, \sigma_{a b}, \mathfrak{W}_{a}{ }^{\mathfrak{a}}$, and $\phi$ are all extensive. Conversely, a variable that is not a function on the phase space of $\Sigma$ is known as an intensive variable. Functions of the lapse and the shift are examples of intesive variables. We see in equation (2) that the first three terms of the integrand involve variations of intensive variables while the last three terms involve variations of extensive variables. We shall return to this important point in the next section.

The quasilocal surface energy, momentum, and charge densities yield valuable information about the enclosed spacetime when it possesses sufficient symmetries. If the boundary, $\mathcal{T}$, admits a timelike, surface forming, Killing vector (such that the Lie derivative of all the fields along this vector vanish), and if the matter stress energy tensor is negligible on the quasilocal boundary, then the quantity $\mathbb{M}=\oint d^{2} x N \mathcal{E}$ (where $\oint$ represents an integral over the quasilocal surface, $\partial \Sigma$ ) is a conserved quantity representing the mass contained within the boundary. By "conserved" we mean that the mass is independent of the foliation of $\mathcal{T}$. Similarly, a conserved angular momentum can be associated with the interior of the boundary under similar conditions, but where the Killing vector, $\varphi^{a}$, must now be spacelike and azimuthal. If the quasilocal boundary contains the orbits of this vector, then the angular momentum is $\mathbb{J}=\oint d^{2} x \mathcal{J}_{a} \varphi^{a}$. Finally, a conserved Yang-Mills charge can be associated with each Lie-algebra-valued scalar field, $\mathfrak{k}^{\mathfrak{a}}$, that is covariantly constant: $\left(\mathfrak{D}_{\mu}\right)^{\mathfrak{a}} \mathfrak{b}_{\mathfrak{b}} \mathfrak{k}^{\mathfrak{b}}=0$. The conserved charge is then $\mathbb{Q}[\mathfrak{k}]=\oint d^{2} x \mathcal{Q}_{\mathfrak{a}} \mathfrak{k}^{\mathfrak{a}}$. This is the analog of Gauss' law. By quasilocal energy, we shall mean the quantity $\mathbb{E}=\oint d^{2} x \mathcal{E}$. Notice that this differs from the quasilocal mass and is not necessarily a conserved quantity. However, it will be interpreted at the thermodynamic internal energy of the system.

\section{The Microcanonical Action}

The action that we have considered thus far is the appropriate action for the generation of the equations of motion when, under variations, the fields on the initial and final spacelike hypersurfaces are fixed as are various quantities on the quasilocal boundary. However, we have seen that the quantities that must be fixed on the quasilocal boundary are a mixture of extensive and intensive variables. The microcanonical action will be that action for which only extensive variables need 
be fixed on the quasilocal boundary under variations. It differs from the original action by a boundary term:

$$
S_{\mathrm{m}}=S+\int_{\mathcal{T}} d^{3} x\left(\mathcal{E} N-\mathcal{J}_{a} N^{a}+\mathcal{Q}_{\mathfrak{a}} N \mathfrak{V}^{\mathfrak{a}}\right)
$$

so that

$$
\delta S_{\mathrm{m}} \mid \mathcal{T}=\int_{\mathcal{T}} d^{3} x N\left(\delta \mathcal{E}-\omega^{a} \delta \mathcal{J}_{a}+\mathfrak{V}^{\mathfrak{a}} \delta \mathcal{Q}_{\mathfrak{a}}+\frac{1}{2} \mathcal{S}^{a b} \delta \sigma_{a b}+\mathcal{I}^{a}{ }_{\mathfrak{a}} \delta \mathfrak{W}_{a}{ }^{\mathfrak{a}}+\mathcal{F} \delta \phi\right)
$$

where $\omega^{a}=N^{a} / N$ is interpreted as the angular velocity of observers with zero vorticity on $\mathcal{T}$. The covariant form of the microcanonical action is just the same as equation (1), but now the $\mathcal{T}$ boundary term is:

$$
\left.S_{\mathrm{m}}\right|_{\mathcal{T}}=\int_{\mathcal{T}} d^{3} x \sqrt{-\gamma}\left(D(\Phi) t_{\mu} \Theta^{\mu \nu} \partial_{\nu} t-2 n^{\mu} \partial_{\mu} D(\Phi)-W(\Phi)\left(n_{\mu} \mathfrak{F}^{\mu \nu}{ }_{\mathfrak{a}} \partial_{\nu} t\right)\left(t^{\mu} \mathfrak{A}_{\mu}{ }^{\mathfrak{a}}\right)\right) .
$$

We now use the microcanonical action to find an expression for the entropy of systems containing a stationary ${ }^{4}$ black hole. Since we will use path integral methods, it will be useful to adopt a "Euclidean" notation. In the Euclideanization prescription, we shall construct a new microcanonical action functional, $I_{\mathrm{m}}=-\mathrm{i} S_{\mathrm{m}}$, so that the phase of the path integral is $\exp \left(-I_{\mathrm{m}}\right)$. The imaginary factor is absorbed into the intensive variables (specifically, the lapse) so that the extensive variables are invariant under the Wick rotation.

The quantum mechanical microcanonical density matrix is defined as the path integral over all fields in $\mathcal{M}$ with the phase weighting $\exp \left(-I_{\mathrm{m}}\right)$. It is a functional of the variables held fixed on the initial and final spatial hypersurfaces, $\Sigma_{\text {initial }}$ and $\Sigma_{\text {final }}$, as well as the extensive variables held fixed on $\mathcal{T}$. The most important statistic of this density matrix is the partition function which, in the microcanonical ensemble, is known as the density of states. This is obtained by identifying the initial and the final spatial hypersurfaces (with some period, $\Delta t$ ) and "tracing over" the field configurations on these identified surfaces. The density of states, then, is a functional of the extensive variables on the qusilocal surface.

Because the system contains a black hole, the foliation becomes degenerate on the event horizon. When the initial and final spatial hypersurfaces have been identified, the complex manifold has the topology of a cone $\times S^{2}$ (we assume that it is posible to foliate from the event horizon to the quasilocal boundary with parameter $r$ ). The conical singularity at the event horizon is removed by fixing the period of identification to the value $\Delta t=2 \pi / \kappa_{\mathrm{H}}$ where $\kappa_{\mathrm{H}}^{2}=h^{i j}\left(\partial_{i} N\right)\left(\partial_{j} N\right)$ (evaluated on the event horizon) is the surface gravity of the event horizon.

We now find the entropy of the gravitating system to "zeroth order" in quantum corrections. In this approximation, the density of states is just the path integral phase evaluated on a classical solution, so the entropy is $\mathbb{S} \approx-\left.I_{\mathrm{m}}\right|_{c \ell}$. We thus need to evaluate the Euclideanized microcanonical action at its (complex) extremal value. The event horizon is not included in the system as it acts as a one-way membrane for observers orbiting on $\mathcal{T}$. This is accoplished most easily by performing a canonical decomposition of the microcanonical action with the surface term given in equation

\footnotetext{
${ }^{4}$ Stationarity of all the fields is a necessary condition of thermodynamic equilibrium.
} 
(5). No boundary term is appended to the action on the event horizon. We find that:

$$
\begin{aligned}
I_{\mathrm{m}}= & \mathrm{i} \int d t\left(\int_{\Sigma} d^{3} x\left(-p^{i j} \dot{h}_{i j}-\mathfrak{P}_{\mathfrak{a}}^{i} \dot{\mathfrak{A}}_{i}^{\mathfrak{a}}-P \dot{\Phi}+\mathcal{H} N+\mathcal{H}_{i} N^{i}+\mathcal{G}_{\mathfrak{a}} \mathfrak{A}_{t}^{\mathfrak{a}}\right)\right. \\
& \left.+\int_{H} d^{2} x\left(2 \sqrt{\sigma}\left(D(\Phi) n^{i} \partial_{i} N-N n^{i} \partial_{i} D(\Phi)\right)-N^{a} \mathcal{J}_{a}+N \mathcal{Q}_{\mathfrak{a}} \mathfrak{V}^{\mathfrak{a}}\right)\right)
\end{aligned}
$$

where the over-dot indicates a Lie derivative with respect to time and $\mathcal{H}, \mathcal{H}_{i}$, and $\mathcal{G}_{\mathfrak{a}}$ are the Hamiltonian, momentum, and Gauss constraints respectively. Since the solution is stationary, all the time derivatives vanish and since the action is to be evaluated on a classical solution, the constraints also vanish. All that is left, then is the integral over the event horizon. Here, we have $N=0, N^{a}=0$, and, from the regularity conditions, $\int d t n^{i} \partial_{i} N=2 \pi \mathrm{i}$. Thus, we find that the entropy is $\mathbb{S}=\int d^{2} x \sqrt{\sigma} D(\Phi)$ where the integral is over the event horizon. Notice that, in the case of General Relativity, we have $D(\Phi)=(16 \pi)^{-1}$, and the entropy is the usual value of one quarter of the event horizon area (in units of the rationalized Planck's constant and Newton's constant).

The first law of thermodynamics follows immediately from equation (4). Define $\beta=\mathrm{i} \int d t N$ evaluated on the quasilocal boundary, $\partial \Sigma$. It is the reciprocal temperature of the system. Notice that it is not necessarily constant over the quasilocal surface unless the quasilocal surface is so chosen. The first law of thermodynamics for the quasilocal system is:

$$
\delta \mathbb{S}=\int_{\partial \Sigma} d^{2} x \beta\left(\delta \mathcal{E}-\omega^{a} \delta \mathcal{J}_{a}+\mathfrak{V}^{\mathfrak{a}} \delta \mathcal{Q}_{\mathfrak{a}}+\frac{1}{2} \mathcal{S}^{a b} \delta \sigma_{a b}+\mathcal{I}^{a}{ }_{\mathfrak{a}} \delta \mathfrak{W}_{a}{ }^{\mathfrak{a}}+\mathcal{F} \delta \phi\right)
$$

When the quasilocal surface is chosen to be an isotherm, the first term of the integral becomes the usual " $\beta \delta \mathbb{E}$ " term. However, since the angular velocity, for example, will not necessarily be constant over an isotherm, it is necessary to write the first law in integral form.

\section{REFERENCES}

Brown, J. D. and York, J. W., Quasilocal energy and conserved charges derived from the gravitational action, Phys. Rev. D. 47 (1993a), 1407-1419.

Brown, J. D. and York, J. W., Microcanonical functional integral for the gravitational field, Phys. Rev. D. 47 (1993b), 1420-1431.

Creighton, J. D. E. and Mann, R. B.. Quasilocal Thermodynamics of Dilaton Gravity Coupled to Gauge Fields, Preprint gr-qc/9505007 (1995).

Department of Applied Mathematics and Theoretical Physics, Silver Street, Cambridge, England. CB3 9EW

Current address: Department of Physics, University of Waterloo, Waterloo, Ontario.

E-mail address: jolien@avatar.uwaterloo.ca, rbm20@amtp.cam.ac.uk 